\title{
Efeitos de uma intervenção sobre a topografia das habilidades sociais de professores 1
}

\author{
Almir Del Prette, Zilda Aparecida Pereira Del ${ }^{1}$ Prette \\ Professores do Departamento de Psicologia da UFSCar
}

Aline Christina Torres e Angela Cristina Pontes

Bolsistas $\mathrm{CNPq}$

\begin{abstract}
Resumo
Examina a influência de um Programa de Desenvolvimento Interpessoal Profissional (PRODIP) sobre aspectos topográficos do desempenho dos professores participantes. A intervenção objetivou desenvolver habilidades interpessoais do professor para o estabelecimento de condições interativas de ensino e foi realizada em doze sessões grupais de hora e meia cada, ao longo de dois meses. Os dados foram coletados através de filmagem de aulas pré e pós-intervenção. A topografia do desempenho dos professores foi descrita e avaliada em seus componentes não verbais, paralíngüísticos e mistos. Os resultados mostraram que todos os professores foram avaliados mais positivamente em vários componentes e que expressão facial, entonação, humor e entusiasmo foram objeto de "melhora" de praticamente todos os professores. Discutem-se esses resultados em relação aos objetivos do PRODIP e a outras questões de pesquisa.

Palavras-Chave: habilidades sociais, formação continuada de professores, comunicação não verbal.
\end{abstract}

\section{Effects of an intervention on the topographic features of teachers' social skills}

\section{Summary}

Present the exame of some effects of an Interpersonal Professional Development Program (PRODIP) concerning to the topographic performance of six teachers that participated in the program. The intervention aimed interpersonal skills required to establish interactive learning conditions. It was completed in twelve sessions of one and a half hour duration, over a two-months period. Each teacher class was videotaped prior to and following the intervention phase. Written descriptions of the topographics features of each theacher's interpersonal behavior were developed and then analysed in terms of its nonverbal, paralinguistic and mixed components. The results indicated that all the teachers were evaluated more positively on several topographic features following the intervention program and that facial expression, entonation and use of humour and entusiasm were improved for nearly all the teachers. These finds are discussed regarding the objectives of the program and other questions research.

Key-Words: social skills, in-service teacher-training, nonverbal communication.

O campo das Habilidades Sociais (HS) vem-se estruturando conceitualmente ao longo dos últimos 30 anos. Sua matriz conceitual é permeada por diferentes modelos (Hidalgo e Abarca, 1992) que contribuíram para sua formação, tanto como abordagem teórica, quanto campo de aplicação. Uma extensa literatura de análise conceitual (Trower, 1980; McFall,1982; Ladd e Mize, 1983; Del Prette \& Del Prette, 1996, entre outros) tem procurado contribuir para uma melhor compreensão da área. O exame dessa literatura evidencia uma grande variedade de definições, que se relacionam aos modelos teóricos adotados.

Não é objetivo deste trabalho fazer uma análise detalhada dos conceitos de HS. Assim, dotamos aqui o conceito de Caballo (1987, p. 14), segundo o qual o comportamento socialmente

\footnotetext{
${ }^{1}$ Este trabalho faz parte do projeto "Interação social e aprendizagem: Habilidades e concepções do professor em uma situação de grupo". financiado pela FAPESP (1995/0690-3. 1995/6940-5. 12995/6132-6), CNPq (520988/95-7 RE) e MEC/SESU (Convênio UFSCar/FNDE 1058/96). Foi originalmente apresentado no III Congresso Nacional de Psicologia Escolar, realizado em novembro de 1996.
} 
habilidoso é "esse conjunto de comportamentos emitidos por um indivíduo no contexto interpessoal, que expressa sentimentos, atitudes, desejos, opiniões ou direitos desse indivíduo de um modo adequado à situação, respeitando esses comportamentos nos demais e que geralmente resolvem uma situação ao mesmo tempo em que minimiza a probabilidade de problemas futuros".

Na análise dessa definição, alguns autores (Caballo, 1987; Caballo, 1991; Del Prette e Del Prette, 1996) apontam para as três dimensões descritivas do constructo habilidades sociais que ela contempla: a) Dimensão pessoal: variáveis e processos encobelios do indivíduo (pensamentos, sentimentos, percepções, expectativas etc.) e também as variáveis fisiológicas (sudorese, batimentos cardíacos, contração/dilatação pupilar etc); b) Dimensão situacional: contexto ambiental em que o desempenho social ocorre (incluindo aspectos culturais); c) Dimensão comportamental: classes de comportamentos abertos do indivíduo.

A dimensão comportamental tem sido considerada, no campo das habilidades sociais, em dois níveis de análise: o dos componentes molares e o dos moleculares. A classificação das ações no nível molecular é sempre relativa ao que está sendo considerado como o nível molecular, ou seja, depende do grau de especificidade e inclusão de uma em relação à outra. No campo das Habilidades Sociais, o termo molecular vem sendo usualmente aplicado às características topográficas do desempenho, entendidas como um dos componentes de sua funcionalidade.

Os componentes molares são as classes funcionais mais amplas de ações e reações dos indivíduos (fazer pedidos, iniciar conversação, expressar desagrado, manter conversação etc), e os componentes moleculares são definidos como os componentes topográficos dessas ações, ou seja, a forma do comportamento. Os componentes moleculares incluem os verbais (conteúdo da fala, uso de perguntas, uso de expressões particulares etc), os não verbais (contato visual, postura, meneios de cabeça, gesticulação etc), os paralingüísticos (volume da voz, entonação, velocidade, clareza etc) e os mistos (autoridade/liderança, humor/formalidade, afetividade etc).

Os componentes moleculares precisam ser coerentes e fortalecedores da mensagem verbal para que esta alcance os efeitos pretendidos sobre o interlocutor. Um adequado desempenho em nível molecular contribui decisivamente para uma percepção de maior competência social (Caballo, 1987; Del Prette e Del Prette, 1997).

A funcionalidade dos componentes moleculares remete à própria funcionalidade das habilidades sociais para produzir as consequiências desejáveis (por exemplo, atingir objetivos imediatos, manter ou melhorar a relação interpessoal e a auto-estima, conforme assinala Linehan, 1984). As pesquisas (Argyle, 1975; 1984; Poyatos, 1983) indicam que a compreensão da comunicação entre os indivíduos depende, em grande parte, dos aspectos não verbais presentes nas interações face-a-face. Enquanto a maioria dos componentes molares são específicos ao contexto em que são emitidos (por exemplo, espera-se do professor a habilidade para organizar uma atividade de grupo em sala de aula e de um político, que tenha habilidade de conversação), a maioria dos componentes moleculares são requeridos em diferentes situações (tanto um professor quanto um político precisam manter um adequado contato visual com o interlocutor ou distribuí-Io para várias pessoas quando falam a um grupo de alunos ou pessoas em geral).

Como os componentes moleculares são usualmente pouco conscientes, a aprendizagem de novos padrões implica exercitar a autopercepção e a automonitoria conscientes, associadas à aprendizagem de padrões molares apropriados, o que, em geral, exige condições estruturadas de ensino ou treinamento. Dado o papel dos componentes moleculares na caracterização do 
desempenho socialmente habilidoso e o fato dessas habilidades serem passíveis de aprendizado, justifica-se a sua inclusão em programas de Treinamento de Habilidades Sociais (THS).

Considerando a importância de interações educativas em sala de aula, para uma efetiva construção social do conhecimento e ressaltando o papel do professor enquanto participante, condutor e mediador dessas interações, defende-se que a competência profissional do professor requer também um repertório altamente diferenciado de habilidades interpessoais que inclui, entre outros aspectos, a percepção das demandas imediatas do contexto escolar, flexibilidade para mudanças na atuação, de acordo com essas demandas, bem como habilidade de produzir conflitos sócio-cognitivos entre os alunos e reagir positivamente às tentativas de solução destes conflitos por parte dos mesmos.

Estudos prévios de Del Prette e Del Prette (1995) mostraram que, embora os professores relatassem emissão de resposta indicativas de um repertório não deficitário de habilidades sociais cotidianas e valorizassem configurações interativas propícias à construção de conhecimento, a sua prática, na maioria das vezes, reservava ao aluno um papel passivo e assimilador.

Ao se considerarem as relações interpessoais como fundamentais para o desenvolvimento e a aprendizagem em contexto escolar, defende-se que o conhecimento produzido no campo teóricoprático das HS pode ser pertinente a estas questões da Psicologia Educacional. De acordo com Del Prette e Del Prette (1997), embora as abordagens construtivistas e sócio-interacionistas derivem-se de posições epistemológicas diferentes daqueles que fundamentam o campo das HS, o desenvolvimento e as tendências mais recentes em HS apontam para uma ênfase crescente em fatores sócio cognitivos e para a possibilidade de explorar seus recursos instrumentais e metodológicos na avaliação e promoção de um repertório de habilidades (interpessoais/cognitivas) importantes ao exercício profissional centrado nas relações interpessoais e intergrupais, como é o caso do magistério.

Considerando-se as questões acima, desenvolveu-se um Programa de Desenvolvimento Interpessoal Profissional (PRODIP), que teve como objetivo geral a promoção da competência interpessoal de professores, instrumentalizando-os para estratégias de ensino que envolvessem interações sociais com e entre os alunos no sentido de maximizar a aprendizagem e 0 desenvolvimento dos mesmos. Essa instrumentalização estava voltada para as habilidades sóciocognitivas do professor em conceber, planejar, conduzir e participar [de] interações sociais educativas em sala de aula e habilidades específicas nelas requeridas, tais como perguntas de maior e menor elaboração, aprovação e feedback aos alunos por esforços e aproximações aos desempenhos requeridos, planejamento e coordenação de atividades de grupo, mediação de tutoria entre alunos, etc. No caso específico das habilidades envolvidas na condução e participação de interações sociais educativas, o PRODIP enfatizou tanto aspectos da funcionalidade como da topografia desse desempenho.

Um dos problemas presentes nos programas de formação continuada refere-se à avaliação de seus efeitos sobre o desempenho dos professores, dadas as dificuldades metodológicas envolvidas na elaboração de procedimentos e critérios de avaliação, especialmente quando se trata do desempenho em sala de aula e, mais precisamente, da transferência de eventuais aquisições para o cotidiano de sua prática pedagógica.

O presente estudo orienta-se por esta preocupação e teve o objetivo específico de examinar a influência do PRODIP sobre o desempenho dos professores em componentes moleculares de sua 
atuação em sala de aula, registrado através de filmagens prévias e posteriores à intervenção. Outras dimensões do desempenho dos professores e outros aspectos do programa estão sendo avaliados em outros estudos.

\section{Método}

\section{Sujeitos}

Seis professores, com idade entre 26 e 47 anos, que se inscreveram no Programa de Desenvolvimento Interpessoal Profissional (PRODIP), realizado em uma escola da rede pública de São Carlos. Os professores possuíam o $3^{\circ}$ grau e ministravam aulas de $5^{\mathrm{a}}$ a $8^{\mathrm{a}}$ séries e/ou $2^{\circ}$ grau: PMI, professora de Inglês, era graduada em Língua Inglesa, com curso de aperfeiçoamento em Inglês; P-RO, professora de Português, era graduada em Língua Alemã e Líteratura Brasileira e mestranda em Literatura; P-PA, professora de Matemática, era graduada em Física e mestranda em Matemática; P-AL, professora de Educação Física, tinha aperfeiçoamento em Metodologia de Ensino; P-NI, professora de Educação Artística, também com aperfeiçoamento em Metodologia de Ensino; PHI, professor de História Geral, com graduação em História.

\section{Contexto da intervenção}

O PRODIP foi conduzido em doze sessões grupais de uma hora e meia cada, ao longo de dois meses. As sessões eram estruturadas previamente em tomo de objetivos de crescente dificuldade (iniciando por habilidades interpessoais mais básicas como as de observação de comportamentos, descrição de situações interpessoais, apresentação de feedback, perguntas, elogios etc, até chegar a habilidades mais complexas como as de coordenar e organizar grupos de discussão em sala de aula, mediar seqüências mais longas de interação com os alunos, responder diferencialmente as verbalizações mais elaboradas dos alunos etc). A estrutura de cada sessão incluía: a) Uma fase inicial de "aquecimento", com jogos e exercícios interpessoais de promoção de habilidades básicas, além de avaliação de aquisições e generalizações para a sala de aula; b) Uma parte central de exposição e discussão conceitual de temas relacionados às áreas de habilidades sociais, desenvolvimento interpessoal, interações sociais de sala de aula, construção social de conhecimento, ensino, aprendizagem etc, além da promoção de habilidades mais complexas; c) Uma parte final de descontração e coesão de grupo, com exercícios vivenciais variados de desenvolvimento da sensibilidade e empatia, além da atribuição de tarefas e avaliação da sessão. A partir das sessões intermediárias, foram introduzidas atividades de videofeedback, onde os professores eram solicitados a analisar e destacar aspectos positivos de trechos de filmagens de sala de aula referentes ao próprio desempenho e desempenho dos colegas em sala de aula.

\section{Procedimento de coleta de dados}

Os dados foram coletados através de filmagens, pré e pós-intervenção, de duas/horas aulas consecutivas (exceto para uma professora que só dispunha de aulas de uma hora). Uma câmera, posicionada no fundo da sala, focalizava o professor e seus interlocutores a cada momento, fechando o ângulo quando o professor se dirigia a alunos específicos e abrindo-o quando se dirigia à classe como um todo.

\section{Procedimento de tratamento dos dados}

Inicialmente, foi feito um exame das filmagens, a partir das imagens, pré-intervenção de três professores, dividindo-se o registro em intervalos de cinco minutos nos quais se processou a 
identificação das subclasses dos componentes moleculares e sua descrição, de acordo com a literatura da área (Caballo, 1987; Caballo \& Buela, 1988; Caballo, 1995). As classes e subclasses próprias dos componentes moleculares das habilidades sociais, selecionadas para análise, são apresentadas no Quadro I, a seguir.

Quadro 1. Classes e subclasses de componentes moleculares examinados nas filmagens

\begin{tabular}{|l|l|l|}
\hline \multicolumn{2}{|c|}{ COMPONENTES MOLECULARES } \\
\hline \multicolumn{1}{|c|}{ NÃO VERBAIS } & \multicolumn{1}{c|}{ PARALINGÜÍSTICOS } & \multicolumn{1}{c|}{ MISTOS } \\
\hline CV - contato visual & VO - volume & AL - autoridade/liderança \\
\hline PO - postura & TI - timbre & HF - humor/formalidade \\
\hline GE - gesticulação & FL - fluidez & EA - entusiasmo/apatia \\
\hline EF - expressão facial & EN - entonação & CA - controle de ansiedade \\
\hline SO - sorriso & VE - velocidade & AF - afetividade \\
\hline ME - meneios de cabeça & CL - clareza & \\
\hline MO - movimentação nela sala & PA - pausas & \\
\hline DP - distância/proximidade & CH - chavões & \\
\hline
\end{tabular}

Com base nessas classes, foi feita a identificação e descrição das subclasses de desempenho molecular dos professores. Em seguida, foram definidos critérios para atribuição de escores para cada subclasse, a partir de discussão entre os pesquisadores considerando-se, de um lado, os aspectos envolvidos na avaliação do padrão molecular das HS (Caballo, 1987) e, de outro, as características de desempenho esperadas de um professor em sala, por exemplo, atenção à classe como um todo e a alunos específicos, fala audível à classe, postura compatível, gestos coerentes com o conteúdo verbal, etc. Para a atribuição de escores, foi utilizada uma escala valorativa de 9 pontos subdivididos em três pólos:

\begin{tabular}{|c|c|c|}
\hline PÓLO & INTER- & PÓLO \\
NEGATIVO & MEDIÁRIO & POSITIVO \\
$1-2-3$ & $4-5-6$ & $7-8-9$ \\
\hline
\end{tabular}

A atribuição de escores foi feita sobre trechos de 2 minutos a cada 10, perfazendo um total de 8 trechos da filmagem pré e 8 da filmagem pós-intervenção (com exceção de P-AL que, filmada em apenas uma aula/hora antes e depois, foi analisada em 4 trechos de 2 minutos a cada 10). Esta avaliação foi realizada por dois observadores independentes, previamente treinados quanto ao ajuste de concordância e critérios para atribuição de escores. Sobre essas duas avaliações, foram calculados os escores médios de cada classe e subclasse para cada professor, organizando-se tabelas e gráficos do desempenho pré e pós-intervenção. Foi estabelecida, como critério de concordância, a pontuação no mesmo pólo (conforme escala acima). Além da atribuição de escores, foi realizada, também, independentemente pelos observadores, uma descrição do padrão molecular de cada subclasse, elaborando-se quadros-resumo descritivos de comparação entre os dados prévios e posteriores à intervenção.

\section{Resultados}

Os dados de resultados foram objeto de duas linhas de análise: as variações no escore e as variações na descrição do padrão molecular entre as medidas pré e pós-intervenção. Os dados relati- 
vos ao escore atribuído ao desempenho molecular de cada um dos participantes do PRODIP são apresentados na Figura 1.

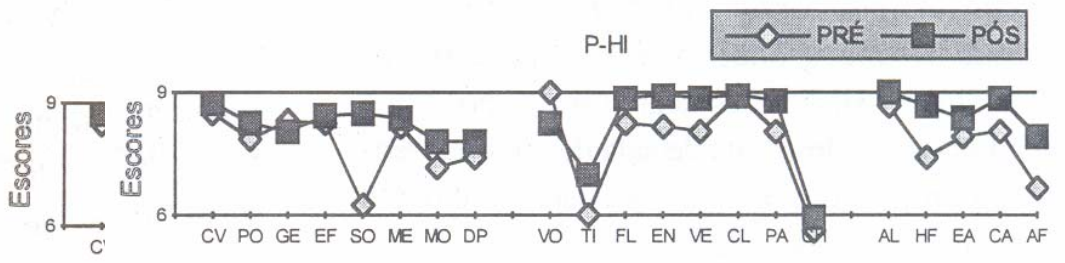

P-MI

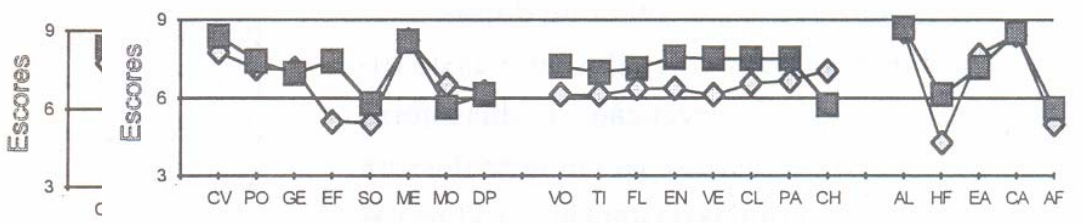

P-RO

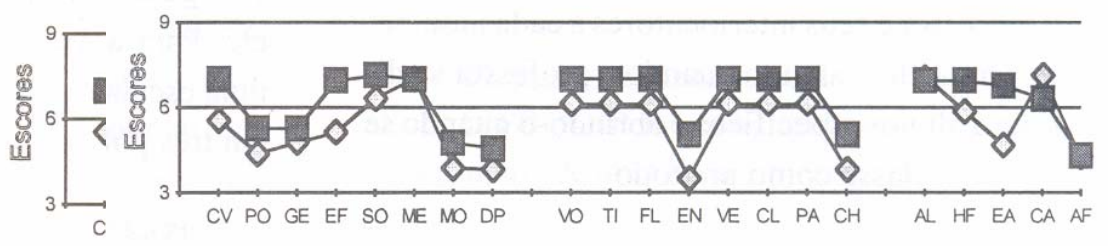

P-AL
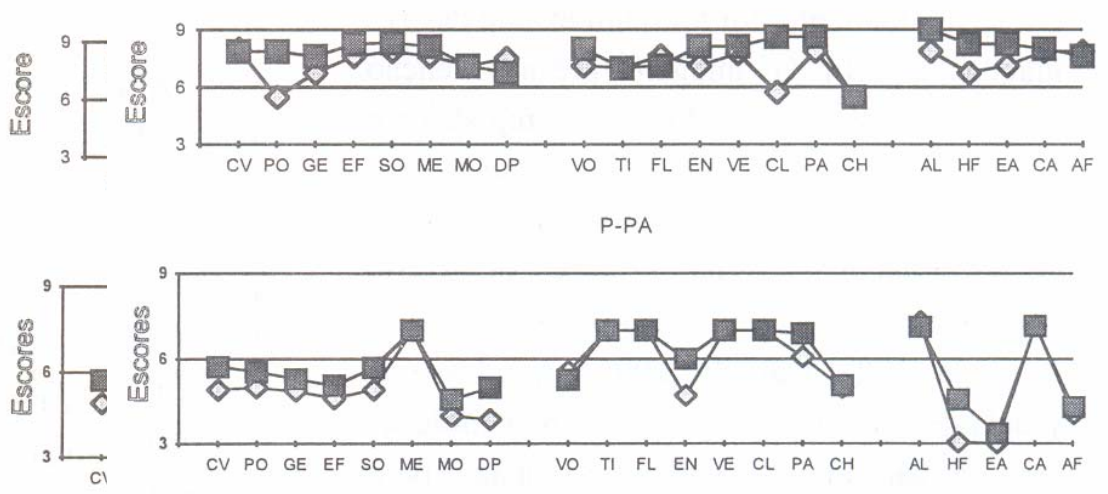

P-NI

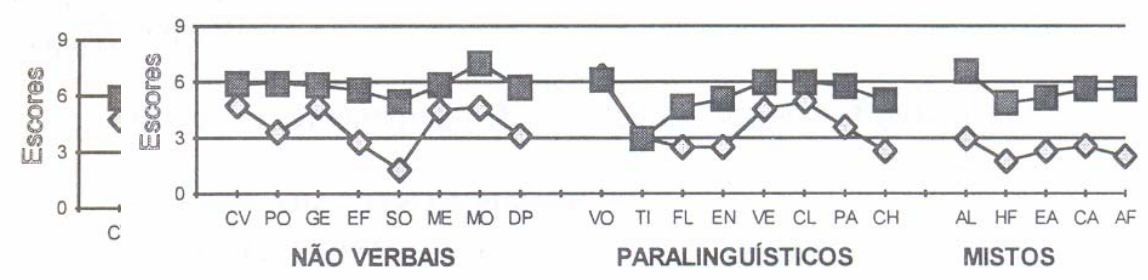

Figura 1. Escores médios atribuídos aos componentes moleculares do desempenho dos professores na avaliação das filmagens pré e pó-intervenção

Na análise da Figura 1, observa-se que, com exceção de PNI, todos os professores foram, já 
na primeira filmagem, avaliados nos pólos intermediário (4-5-6) ou positivo (7-8-9).

Considerando-se uma variação igualou superior a um ponto entre as avaliações pré e pósintervenção como indicativo de mudança no desempenho molecular, verifica-se que:

a) P-NI passou do pólo negativo para o intermediário em 14 subclasses (PO, EF, SO, DP, TI, FL, EN, PA, CH, AL, HF, EA, CA e AF);

b) P-HI passou do pólo intermediário para o positivo em 3 subclasses (SO, TI e AF), P-AL em 2 subclasses (PO e HF), P-MI em 2 subclasses (EF e EN) e P-RO em 1 (CV);

c) P-MI apresentou mudança dentro do pólo intermediário em 7 subclasses (CV, GE, ME, MO, VO, VE e CL) P-RO em 5 (EF, CH, EN, HF e EA), P-P A em 3 subclasses (DP, EN e HF), e P-MI em 1 subclasse (HF);

d) P-AL apresentou melhora dentro do pólo positivo em 3 subclasses (EN, AL e EA) e P-HI em 1 subclasse (HF);

e) P-PA não apresentou mudança de pólo em nenhuma subclasse e P-NI apenas em 1 subclasse (VO);

f) P-MI apresentou desempenho contrário aos objetivos do PRODIP na subclasse $\mathrm{CH}$, passando do pólo positivo para o pólo intermediário.

Um resumo das subclasses em que os professores apresentaram melhoras no desempenho molecular é apresentado no Quadro 2.

Examinando o Quadro 2, observa-se que P-NI, avaliada nas medidas pré-intervenção predominantemente no pólo negativo, foi o professor que apresentou melhora em maior número de subclasses. Nota-se, ainda, que as subclasses HF, EN, EF e EA foram aquelas em que maior número de professores apresentaram melhoras e a subclasse VO não foi avaliada como foco de melhora para nenhum professor. Na subclasse $\mathrm{CH}$, em P-MI, houve mudança em sentido contrário aos objetivos do PRODIP.

Quadro 2. Indicações das classes e subclasses de componentes moleculares em que os participantes do PRODIP apresentaram melhoras entre as medidas pré e pós-intervenção

\begin{tabular}{|c|c|c|c|c|c|c|c|c|c|c|c|c|c|c|c|c|c|c|c|c|c|c|}
\hline \multicolumn{10}{|c|}{ NÃO VERBAIS } & \multicolumn{10}{|c|}{ P ARALINGÜÍSTICOS } & \multicolumn{1}{c|}{ MISTOS } \\
\hline PROF & CV & PO & GE & EF & SO & ME & MO & DP & VO & TI & FL & EN & VE & CL & PA & CH & AL & HF & EA & CA & AF & TOTAL \\
\hline P-MI & & & & $\mathrm{X}$ & & & & & & & & $\mathrm{X}$ & & & & & & $\mathrm{X}$ & & & & 3 \\
\hline P-RO & $\mathrm{X}$ & & & $\mathrm{X}$ & & & & & & & & $\mathrm{X}$ & & & & $\mathrm{X}$ & & $\mathrm{X}$ & $\mathrm{X}$ & & & 6 \\
\hline P-PA & & & & & & & & $\mathrm{X}$ & & & & $\mathrm{X}$ & & & & & & $\mathrm{X}$ & & & & 3 \\
\hline P-AL & & $\mathrm{X}$ & & & & & & & & & & $\mathrm{X}$ & & & & & $\mathrm{X}$ & $\mathrm{X}$ & $\mathrm{X}$ & & & 5 \\
\hline P-NI & $\mathrm{X}$ & $\mathrm{X}$ & $\mathrm{X}$ & $\mathrm{X}$ & $\mathrm{X}$ & $\mathrm{X}$ & $\mathrm{X}$ & $\mathrm{X}$ & & $\mathrm{X}$ & $\mathrm{X}$ & $\mathrm{X}$ & $\mathrm{X}$ & $\mathrm{X}$ & $\mathrm{X}$ & $\mathrm{X}$ & $\mathrm{X}$ & $\mathrm{X}$ & $\mathrm{X}$ & $\mathrm{X}$ & $\mathrm{X}$ & 20 \\
\hline P-HI & $\mathrm{X}$ & & & & $\mathrm{X}$ & & & & & $\mathrm{X}$ & & & & & & & & $\mathrm{X}$ & & & $\mathrm{X}$ & 4 \\
\hline
\end{tabular}

* Ver códigos-especificados no Quadro 1

A análise descritiva do padrão molecular, contraposta à análise quantitativa em termos de escores atribuídos aos professores nas diferentes subclasses, permitiu identificar três grupos de dados apresentados a seguir: A) Mudanças tanto nos escores como na análise descritiva; B) Mudanças nos escores que não se refletiram na análise descritiva; C) Mudanças na análise descritiva não contempladas nos dados de escore. As subclasses e professores associados a cada um 
desses subconjuntos são apresentados no Quadro 3.

Quadro 3. Indicação das subclasses conforme coerência entre alterações anos escores e na descrição efetuada

(A: mudança em ambos; B: mudança nos escores que não se refletiram na descrição e C: mudanças na descrição que não se refletiram nos escores)

\begin{tabular}{|l|l|l|c|c|c|c|c|c|c|c|c|c|c|c|c|c|c|c|c|c|c|}
\hline \multicolumn{10}{|c|}{ NÃO VERBAIS } & \multicolumn{10}{|c|}{ ARALINGÜÍSTICOS } & \multicolumn{7}{c|}{ MISTOS } \\
\hline PROF & CV & PO & GE & EF & SO & ME & MO & DP & VO & TI & FL & EN & VE & CL & PA & CH & AL & HF & EA & CA & AF \\
\hline P-MI & & & & A & & & & & & & & A & & & & & & & A & & \\
\hline PoRO & A & & & A & & & & & & & & B & & & & & A & & B & B & \\
\hline PoPA & C & & & C & & & A & & & & A & & & & C & & C & A & & \\
\hline P-AL & & & & & & & & A & & & & AB & & & & & & & AB & B & \\
\hline P-NI & A & A & A & A & A & A & A & A & & A & A & A & A & A & A & A & A & A & A & A & A \\
\hline P-HI & & & & & A & & C & & & B & & & & & & & & & B & C & A \\
\hline
\end{tabular}

* Ver códigos especificados no Quadro I

O grupo A, que apresentou mudanças tanto nos escores como na análise descritiva, pode ser exemplificado (Quadro 4) com a descrição de algumas subclasses de componentes moleculares de P-NI, a professora que apresentou maior variação entre os escores pré e pós-intervenção.

Quadro 4. Descrição comparativa dos componentes moleculares de P-NI nos momentos pré e pós intervenção

\begin{tabular}{|c|l|l|}
\hline COMPONENTES & \multicolumn{1}{|c|}{ PRÉ-INTER VENÇÃO } & \multicolumn{1}{|c|}{ PÓS-INTERVENÇÃO } \\
\hline SORRISO & Mantém expressão séria, raramente sorrindo & $\begin{array}{l}\text { Sorri em alguns momentos, coerentemente com } \\
\text { o conteúdo da fala e o contexto, para a sala e } \\
\text { para alunos específicos; nos momentos em que } \\
\text { não sorri, mantêm expressão serena. }\end{array}$ \\
\hline $\begin{array}{c}\text { DIST ÂNCIAI } \\
\text { PROXIMIDADE }\end{array}$ & $\begin{array}{l}\text { Permanece distante dos alunos; em alguns } \\
\text { momentos inclina-se na direção de alguns; } \\
\text { raramente os toca; quando o faz, toca-os } \\
\text { rapidamente, raramente com contatos visual. }\end{array}$ & $\begin{array}{l}\text { Alterna aproximação e distanciamento dos } \\
\text { alunos nos diferentes pontos da sala, } \\
\text { movimentando-se constantemente pelo } \\
\text { ambiente; quando solicitada pelos alunos } \\
\text { aproxima-se. }\end{array}$ \\
\hline ENTONAÇÃO & $\begin{array}{l}\text { Mantém a mesma entonação nos diferentes } \\
\text { momentos de aula. }\end{array}$ & $\begin{array}{l}\text { Altera a entonação em alguns momentos, para } \\
\text { enfatizar o conteúdo de suas verbalizações. }\end{array}$ \\
\hline $\begin{array}{c}\text { AUTORIDADE/ } \\
\text { LIDERANÇA }\end{array}$ & $\begin{array}{l}\text { Raramente obtém silêncio e atenção dos alu- } \\
\text { nos enquanto expõe a atividade; obtém parti- } \\
\text { cipação dos alunos, porém com pouco } \\
\text { envolvimento deste na atividade; raramente } \\
\text { obtém resposta às perguntas feitas para a } \\
\text { classe como um todo. }\end{array}$ & $\begin{array}{l}\text { Na maioria das vezes obtém atenção e silêncio } \\
\text { enquanto está expondo um conteúdo ou dando } \\
\text { coordenadas da atividade; obtém resposta à } \\
\text { maioria das perguntas feitas à classe como um } \\
\text { todo; obtém participação/envolvimento da } \\
\text { maioria dos alunos na atividade proposta. }\end{array}$ \\
\hline
\end{tabular}

O grupo B (Quadro 3), cujas mudanças nos escores não se refletiram na análise descritiva, pode ser exemplificado com a avaliação da entonação de P-AL e do timbre de P-HI, subclasses avaliadas com escores mais favoráveis na filmagem pós-intervenção. A entonação de P-AL foi descrita, em ambos os momentos, como adequada, variada e adaptando-se ao seu conteúdo verbal. A descrição do entusiasmo/apatia de P-RO, com escore mais alto na avaliação pós - intervenção, menciona seu envolvimento, interesse e entusiasmo pela atividade e pelo exercício de seu papel em 
sala de aula, tanto nas descrições pré quanto pós-intervenção.

No grupo C (Quadro 3), que apresentou mudanças na análise descritiva não contempladas nos dados de escore, pode-se citar as subclasses movimentação, para P-HI e autoridade/liderança, para P-PA, ambas com escore pré-semelhante ao escore pós, porém com descrições que justificariam uma alteração positiva nesses escores. Na análise da filmagem prévia à intervenção, descreve-se a movimentação de P-HI em frente e no centro da sala, sem qualquer aproximação para a fileira da esquerda; nas descrições pós-intervenção, uma movimentação por toda a sala, de forma freqüente e espontânea. A autoridade/liderança de P-PA foi descrita, nas medidas pré-intervenção, como obtendo atenção, participação e envolvimento da maioria dos alunos através de perguntas, embora não esperasse pelas respostas; na descrição pós-intervenção, a descrição faz referência à alteração em parte desse padrão, registrando que professor induz a participação dos alunos através de perguntas e faz pausas para os alunos elaborarem as respostas.

Neste terceiro grupo (C), a análise descritiva de três subclasses (MO em P-MI, CA e AL em P-RO) revelou mudanças em direção contrária aos objetivos do PRODIP, embora os escores tenham permanecido aproximadamente os mesmos nas avaliações pré e pós-intervenção. Por exemplo, no caso do controle de ansiedade de P-RO, tanto as descrições pré como pós-intervenção faziam referência à sua calma e tranqüilidade, diante dos eventuais incidentes perturbadores de sala de aula, porém, na segunda descrição, há referência a momentos em que a professora reage com aumento no tom de voz e com bater palmas ou a mão sobre a carteira para pedir silêncio. Contudo deve-se salientar que estes três itens representam apenas $4 \%$ da combinação professor/componentes examinada.

\section{Discussão}

Uma visão geral dos resultados permite afirmar que estes ocorreram no sentido de consecução dos objetivos do PRODIP, uma vez que todos os professores foram avaliados mais positivamente em algumas subclasses e algumas subclasses foram objeto de "melhora" de todos ou quase todos os professores (expressão facial, entonação, humor/formalidade e entusiasmo/apatia). A ocorrência dessas alterações se torna ainda mais significativa quando se verifica que a maioria dos professores já havia sido pontuada, nas medidas pré-intervenção nos pólos positivo e intermediário (tendo, portanto, uma menor amplitude para mudanças em direção ao pólo positivo máximo da escala). Esse foi o caso principalmente de P-HI e P-MI, que apresentaram as avaliações e descrições mais positivas nas filmagens pré-intervenção.

Esses resultados podem ser relacionadas, em grande parte, às condições estabelecidas no PRODIP, onde os coordenadores estimulavam os professores, mesmo aqueles com desempenho satisfatório, a elaborarem ainda outras alternativas de atuação, valorizando uma maior flexibilidade comportamental.

As alterações positivas mais evidentes no escore foram observadas no desempenho de PNI, a professora com mais déficits nas medidas pré-intervenção. Uma explicação para isto pode estar nas suas próprias dificuldades iniciais, pois era a professora do grupo que mais tinha a mudar. Em segundo lugar, os demais participantes do PRODIP, com desempenhos já satisfatórios, podem ter-se transformado em modelos para essa professora. A ênfase do PRODIP no uso de feedbacks positivos deve ter tornado mais salientes e mais valorizados esses modelos. O clima não coercitivo do grupo, onde os aspectos negativos do desempenho dos participantes eram, tanto quanto possível, ignorados 
pelos coordenadores e os positivos, altamente valorizados, enfatizando-se a observação e a descrição desses desempenhos, pode ter contribuído para que a professora adquirisse uma melhor percepção de suas dificuldades e buscasse mais ativamente alternativas de desempenhos diferenciadas e adequadas.

As observações informais, ao longo do PRODIP permitiram verificar uma crescente motivação dos participantes, detectada na subclasse entusiasmo/apatia, expressão facial e entonação. Pode-se relacionar esses resultados à importância do humor e de vários componentes não verbais e paralinguísticos, valorizados ao longo do PRODIP.

A análise descritiva complementou os dados quantitativos, confirmando-os em $89 \%$ da combinação itens/professores. Uma hipótese para as exceções pode relacionar-se ao caráter exploratório do procedimento de análise, que se encontra ainda em processo de refinamento. Estas exceções repetiram-se para as mesmas três subclasses (entonação, humor/formalidade e entusiasmo/apatia) e para apenas dois professores (P-RO e P-AL). No caso de humor/formalidade e entusiasmo/apatia, pode-se relacionar a dificuldade de uma maior precisão à própria natureza dessas subclasses que, enquanto componentes mistos, requerem uma análise mais ampla e contextual, sendo mais facilmente "contaminados" por avaliações positivas nos demais componentes moleculares, dificultando uma avaliação mais fidedigna e restrita à própria definição da subclasse. A avaliação dos componentes paralingüísticos, particularmente a entonação, torna-se bastante complexa quando se enfatizam, na definição, os aspectos da variação e da modulação como desejáveis. De qualquer modo, é possível um aperfeiçoamento na definição, através de critérios que possam ajustar-se às modificações na entonação. Tais critérios poderiam ser fornecidos por especialistas da área de fonação.

Quanto às mudanças no padrão descrito que não se refletiram nos escores, uma hipótese formulada é a de que a descrição foi temporalmente mais delimitada (a intervalos de dois minutos), enquanto a avaliação por escore baseou-se no conjunto das descrições, estando, portanto, mais sujeita a um efeito de gestalt, ou seja, a uma generalização entre as avaliações feitas nos intervalos menores. Outra hipótese, ainda, é a de que tais mudanças possam refletir aspectos mais sutis que, embora não considerados na atribuição dos escores, foram objetos de descrição em alguns dos intervalos examinados, indicando, de todo modo, a necessidade de especificar ainda mais os critérios de avaliação.

Um caminho a ser seguido poderia ser o de descrever e avaliar as classes moleculares em termos de sua adequação às classes molares específicas das quais elas são componentes, uma alternativa que está sendo encaminhada pelos autores, como forma de se obter uma avaliação mais completa e compreensiva do desempenho de cada professor e de se examinar mais precisamente o papel destes componentes moleculares na caracterização do comportamento (molar) habilidoso. Outra hipótese para as discrepâncias entre a análise descritiva e a atribuição de escores é a de que uma "piora" nos componentes moleculares possa representar apenas uma transição (ensaios, muitas vezes, exagerados) para formas mais elaboradas e harmoniosas de desempenho. Em outras palavras, é razoável supor que as tentativas iniciais do professor em promover interações mais educativas (nível molar de análise) possam causar-lhe, inicialmente, um aumento de ansiedade (situação nova) que se reflita, de imediato, em "excessos" nos componentes moleculares, embora estes se representem passos na direção dos desempenhos estabelecidos como objetivos do PRODIP. Em apoio a essa hipótese, a literatura tem mostrado que, em vários dos componentes moleculares, tanto 
os extremos de excesso como os de falta são indesejáveis. (Caballo, 1995; Del Prette e Del Prette, 1997). Para exemplificar, pode-se citar a "piora" no controle de ansiedade de P-RO. Outro exemplo é o desempenho de P-MI, que apresentou melhora na subclasse humor/formalidade, tornando-se mais descontraída e informal, o que, por outro lado, pode ter contribuído para o aumento excessivo no uso de chavões, prejudicando sua avaliação nesta subclasse (conforme os critérios adotados na presente avaliação).

Relacionando-se os resultados obtidos às diferentes áreas de conhecimento em que atuavam os professores participantes do PRODIP, pode-se levantar a hipótese de que algumas dessas áreas sejam, por seus próprios objetivos, mais orientadas para a interação social, à comunicação, à criatividade do que para o desenvolvimento cognitivo e intraindividual. No primeiro caso, poderíamos colocar a Comunicação e Expressão (Língua Portuguesa), o Inglês (línguas em geral) e a Educação Artística. No entanto os dados mostram que a professora com maiores déficits iniciais era a de Educação Artística e um dos professores com menor dificuldade era o de História. De todo modo, a hipótese de que características interpessoais do professor estejam associadas às demandas próprias das disciplinas por ele ministradas parece constituir-se em uma questão empírica.

Concluindo, pode-se afirmar que os resultados apresentados constituem indicadores da efetividade do PRODIP em promover melhoras nos componentes moleculares do desempenho dos professores, embora afirmações sobre o significado dessas melhoras na direção de maior competência interpessoal/profissional para conduzir interações sociais educativas ainda requeiram análises adicionais. No entanto se, de fato, como a literatura atesta, os componentes moleculares são importantes para a percepção de competência social, as melhoras, no presente caso, são indispensáveis, embora certamente não suficientes, para as habilidades interpessoais-profissionais do professor.

\section{Referências Bibliográficas}

Argyle, M. (1975). Bodyly communication. London: Methuen.

Argyle, M. (1984). Psicologia dei comportamiento interpersonal. Madri: Alianza Unversidad.

Caballo, V. E. \& Buela, G. (1988). Molecular/molar assessment in an analogue situatcion: relationships among several measures and vaJidation ofa behavioral assessment instruments. Perceptual and Motor Skills, 67, 591-602.

Caballo, V. E. (1991). EI entrenamiento en habilidades sociales. In V. E. Caballo (Ed.) (Org.), Terapia y Modificacion de Conducta (pp. 403-443). Madrid: Siglo Veintiuno.

Caballo, V. E. (1995). Una aportación espafiola aios aspectos moleculares, a la evaluación y ai entrenamiento de las habilidades sociales. Revista Mexicana de Psicologia, /2(2), 121-131.

Caballo, V. E. (Ed.)(1987). Teoria. evaluaciónyentrenamiento de Ias habilidades sociales. Valencia: Promolibro.

Del Prette, A. e Del Prette, Z. A. P. (1997). Habilidades sociais e construção de conhecimento em contexto escolar. In D. R. Zamignani (Org.), Sobre comportamento e cognição (vol. 3, capo 30, 234-250). Santo André (SP): ARBytes.

Del Prette, Z. A. P. \& Del Prette, A. (1995). Interações sociais em sala de aula: representações do professor. In R. Guzzo, G. P. Witter, S. Pfromm Netto, E. Rosado \& S. Wechsler (Orgs.), O futuro da criança na escola,familia e sociedade (pp. 426-430). São Paulo: Átomo. 
Del Prette, Z. A. P. \& Del Prette, A. (1996). Habilidades Sociais: uma área em desenvolvimento. Psicologia: Reflexão e Critica, 9 (2), 287-309.

Hidalgo, C. H. \& Abarca, N. M. (1992). Comunicacion interpersonal- Programa de entrenamiento en habilidades sociales. Santiago do Chile: Editorial Universitaria.

Ladd, W. G. \& Mize, J. (1983). A cognitive-social learning model of social skill training. Psychological Review, 90, 127-157.

Linehan, M. M. (1984). Interpersonnal effectiveness in assertive situations. In: E. E. Bleechman (Ed.), Behavior modification with women. New York: Guilford Press.

McFall, R. M. (1982). A review and reformulation of the concept of social skills. Behavioral Assessment,4, 1-33.

Poyatos, F. (1984). New perspectives in nonverbal communication: Studies in cultural anthropology, social psychology, linguistics, literature and semiotics. New York: Pergamon Press.

Trower, P. (1980). Situatinal analysis ofthe components and processes ofbehavior of social skilled and unskilled patients. Journal ofConsulting and clinícal Psychology, 48,327-329. 